\title{
Ursolic acid inhibits early lesions of diabetic nephropathy
}

\author{
YI ZHOU ${ }^{1}$, JIAN-SHUANG LI $^{1}$, XIN ZHANG ${ }^{2}$, YA-JUN WU ${ }^{1}$, KUN HUANG $^{2}$ and LING ZHENG $^{1}$ \\ ${ }^{1}$ College of Life Sciences, Wuhan University, Wuhan 430072; ${ }^{2}$ School of Pharmacy, \\ Huazhong University of Science and Technology, Wuhan 430030, P.R. China
}

Received May 6, 2010; Accepted June 28, 2010

DOI: 10.3892/ijmm_00000500

\begin{abstract}
The present study sought to investigate the effects of ursolic acid (UA) on the development of glomerular hypertrophy and type IV collagen accumulation, two early lesions associated with diabetic nephropathy (DN). By treating streptozotocin (STZ)-induced diabetic mice with low-dose UA $(0.01 \%$ in food) for three months, the diabetes-induced glomerular hypertrophy and type IV collagen accumulation in the kidneys were found to be markedly ameliorated. Further studies identified that UA treatment suppressed diabetesinduced activations of STAT-3, ERK1/2 and JNK pathways, but not the diabetes-induced activation of the p38 pathway. Furthermore, diabetes-induced overexpression of iNOS in the renal cortex was also significantly suppressed by the treatment. UA may thus be considered as a potential therapeutic agent in treating DN.
\end{abstract}

\section{Introduction}

Diabetic nephropathy (DN), a diabetic microvascular complication, is the leading cause of chronic kidney failure and end stage renal disease in diabetic patients. Glomerular hypertrophy and excellular matrix protein accumulation are key early diabetic nephropathy lesions contributing to the development of final renal failure $(1,2)$. Pathways that include elevated reactive oxygen species, altered MAPK (mitogenactivated protein kinase) pathways and the JAK-STAT (Janus kinase-signal transducer and activator of transcription)

Correspondence to: Dr Ling Zheng, College of Life Sciences, Wuhan University, Wuhan 430072, P.R. China

E-mail: lzheng217@hotmail.com

Dr Kun Huang, School of Pharmacy, Huazhong University of Science and Technology, Wuhan 430030, P.R. China

E-mail: kunhuang2008@hotmail.com

Abbreviations: DN, diabetic nephropathy; iNOS, inducibe nitric oxide synthase; JAK, Janus kinase; MAPKs, mitogen-activated protein kinases; PASH, periodic acid-schiff and hematoxylin; STAT, signal transducer and activator of transcription; UA, ursolic acid

Key words: diabetic nephropathy, uroslic acid, STAT3, MAPKs pathway (3-5) have been suggested to play important roles in the development of DN. Accumulating evidence suggests that pharmacological compounds capable of normalizing these pathways may be used to inhibit the development of the disease (3-5).

Ursolic acid (UA) is a triterpene compound derived from the berries, fruits, leaves and flowers of many medicinal plants. It has been used in Asia for centuries as an antitumor, antiinflammatory, anti-hyperglycemia and immunomodulatory drug $(6,7)$, however, the exact molecular mechanism underlying its beneficial effects remain unclear. A recent study reported that administering high doses of UA and its isomer oleanolic acid $(0.1 \%$ and $0.2 \% \mathrm{w} / \mathrm{w}$ in food $)$ resulted in anti-glycative effects in the kidneys of diabetic mice (8), possibly due to the hypoglycemic effects associated with the administration of high doses of UA. To study the molecular mechanisms underlying UA's beneficial effects on $\mathrm{DN}$, in the present study, UA was administered to insulin-deficient diabetic mice at a much lower dose $(0.01 \% \mathrm{w} / \mathrm{w}$ in food $)$. Experiments were carried out to study 3 aspects of the effects of UA on DN: a) early DN lesions including glomerular hypertrophy and excellular matrix protein accumulation; b) signaling pathways involved in the development of DN, including STAT and MAPK pathways; and c) the expression level of inducibe nitric oxide synthase (iNOS), an inflammatory enzyme.

\section{Materials and methods}

Streptozotocin (STZ)-induced diabetes model. Male C57BL mice were obtained from the ABSL-III laboratory of Wuhan University and housed in a temperature-controlled and lightcontrolled environment with free access to water and food. Insulin-deficient diabetes was induced as we previously reported $(9,10)$. The diabetic mice were randomly divided into two groups: diabetics $(n=10)$ and diabetics with UA treatment $(0.01 \% \mathrm{w} / \mathrm{w}$ in food; $\mathrm{n}=10)$. Mice $(\mathrm{n}=10)$ of similar age injected with citrate buffer were used as nondiabetic controls. Animals were handled according to the guidelines approved by the Committee on Ethics in the Care and Use of Laboratory Animals of Wuhan University. Three months after onset of diabetes, blood glucose levels of experimental mice were measured again to confirm the diabetic conditions before they were sacrificed.

Histology. The kidneys from experimental animals were rapidly dissected and fixed in $10 \%$ buffered formalin at $4^{\circ} \mathrm{C}$ 
Table I. Characteristics of experimental mice.

\begin{tabular}{|c|c|c|c|c|}
\hline Group (n) & $\begin{array}{l}\text { Body weight } \\
(\mathrm{g})\end{array}$ & $\begin{array}{c}\mathrm{NFBG} \\
(\mathrm{mmol} / \mathrm{dl})\end{array}$ & $\begin{array}{c}\text { Kidney weight } \\
(\mathrm{mg})\end{array}$ & $\begin{array}{l}\text { Kidney weight/ } \\
\text { body weight }(\%)\end{array}$ \\
\hline SD (10) & $22.9 \pm 1.6^{\mathrm{a}}$ & $27.2 \pm 5.6^{\mathrm{a}}$ & $178.6 \pm 19.6^{\mathrm{a}}$ & $0.78 \pm 0.10^{\mathrm{a}}$ \\
\hline SD+UA (10) & $24.2 \pm 1.2^{\mathrm{a}}$ & $23.0 \pm 4.4^{\mathrm{a}}$ & $177.6 \pm 14.4^{\mathrm{a}}$ & $0.74 \pm 0.07^{\mathrm{a}}$ \\
\hline $\mathrm{N}(10)$ & $27.5 \pm 2.3$ & $11.2 \pm 1.4$ & $163.8 \pm 20.3$ & $0.60 \pm 0.07$ \\
\hline
\end{tabular}

NFBG, non-fasting blood glucose; SD, diabetes; SD+UA, diabetes treated with ursolic acid; N, nondiabetes. ${ }^{\text {a }} \mathrm{p}<0.05$ vs. nondiabetic group.

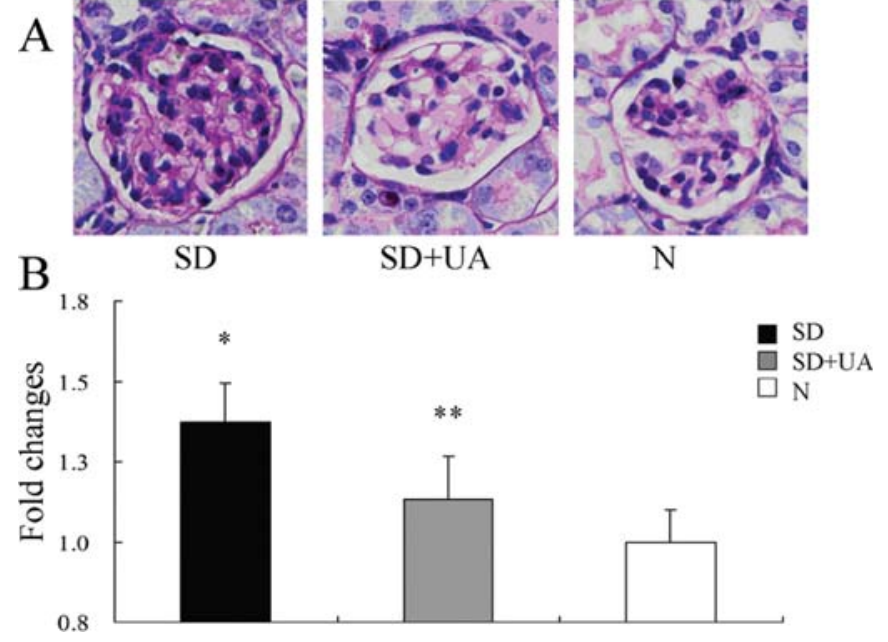

Figure 1. UA inhibits diabetes-induced glomerular hypertrophy. (A) Representative PASH stained kidney sections. (B) Quantification measurement results of glomerular volume. The data are reported as fold changes that normalized to nondiabetic group, which was set up as $100 \%$. ( $\mathrm{SD}$, diabetes, $\mathrm{n}=10 ; \mathrm{SD}+\mathrm{UA}$, diabetes treated with UA, $\mathrm{n}=10 ; \mathrm{N}$, nondiabetes, $\mathrm{n}=10 ;{ }^{*} \mathrm{p}<0.05$ vs. nondiabetic group; ${ }^{* *} \mathrm{p}<0.05$ vs. diabetic group).

overnight. The kidneys were embedded in paraffin followed by sectioning at $5-\mu \mathrm{m}$ thickness on positively charged slides. Sections were stained with periodic acid-schiff and hematoxylin (PASH) as we previously described (11). High resolution pictures of 25-35 glomeruli per animal were taken under an Olympus BX60 microscope equipped with a digital CCD (x400 power), and the glomerular histology was examined in a masked manner. The glomerular cross-sectional areas (Ag) were measured using the ImagePlus 6.0 software (Media Cybernetics, Bethesda, MD). The glomerular volume $(\mathrm{Vg})$ was calculated as described (12) using the relation that $\mathrm{Vg}=\beta / \mathrm{k}[\mathrm{Ag}]^{3 / 2}$, and was further normalized to the mean volume (set as 1) of the nondiabetic group.

Immunohistochemical detection of type IV collagen. Paraffinembedded sections were deparaffinized in xylene and rehydrated in decreasing concentrations of ethanol. Sections were incubated with $3 \% \mathrm{H}_{2} \mathrm{O}_{2}$ for 5 min to quench endogenous peroxidase activity followed by blocking with $2 \%$ goat serum in PBS and immunostained with anti-type IV collagen antibody (Rockland, Gilbertsville, PA) at $4^{\circ} \mathrm{C}$ overnight. After extensive washing with PBST, sections were incubated with

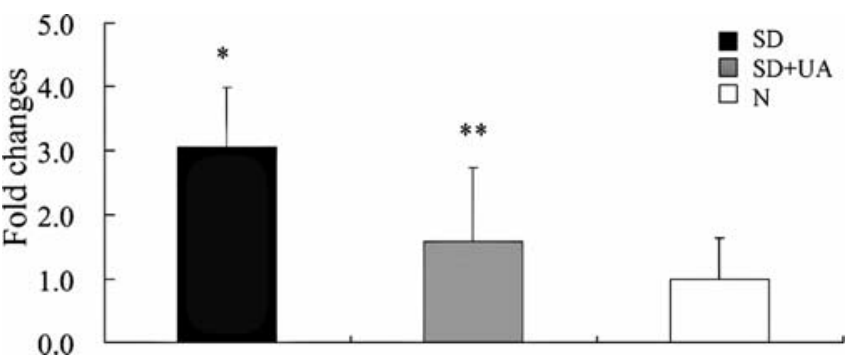

Figure 2. UA inhibits diabetes-induced increase in type IV collagen accumulation in glomeruli. Semi-quantification analysis of type IV collagen staining in the kidneys. The data are reported as fold changes that normalized to nondiabetic group, which was set up as one-fold. (SD, diabetes, $\mathrm{n}=10 ; \mathrm{SD}+\mathrm{UA}$, diabetes treated with UA, $\mathrm{n}=10 ; \mathrm{N}$, nondiabetes, $\mathrm{n}=10$; ${ }^{*} \mathrm{p}<0.05$ vs. nondiabetic group; ${ }^{* *} \mathrm{p}<0.05$ vs. diabetic group).

biotinylated anti-rabbit antibody (Vector Laboratories, Burlingame, CA) for $1 \mathrm{~h}$ at room temperature. Positive staining was visualized by DAB substrate (Vector laboratories) reaction following the $\mathrm{ABC}$ kit (Vector laboratories). High resolution pictures of 25-35 glomeruli per animal were taken under an Olympus BX60 microscope equipped with a digital CCD (x400 power). Semi-quantitative analysis of type IV collagen (scale: 0-4) was performed by two investigators blinded to the samples. The data are reported as fold changes with the nondiabetic group set as 1 .

Western blot analysis. The renal cortex was dissected under a microscope and frozen at $-80^{\circ} \mathrm{C}$ until use. Cortex tissue $(20 \mathrm{mg})$ was sonicated in RIPA buffer (Beyotime Biotech, China). The amount of total protein was determined using the BCA protein assay (Beyotime Biotech). Samples (20-50 $\mu \mathrm{g}$ protein/ sample) were separated by SDS-PAGE and electroblotted onto nitrocellulose membrane (Bio-Rad, Hercules, CA). Membranes were stained with washable Ponceau S solution to visualize the equal protein loading. After a brief wash, the membranes were blocked in $2 \%$ nonfat milk (Bio-Rad). Primary antibodies against total STAT-3, phosphorylated STAT-3, total ERK1/2, phosphorylated ERK1/2, total p38 MAPK, phosphorylated p38 MAPK, total JNK, phosphorylated JNK (1:1000 dilution; Cell Signaling Technology, Danvers, MA), and iNOS (1:1000 dilution; BD Biosciences, San Jose, CA) were applied overnight at $4^{\circ} \mathrm{C}$. After extensive washing, all blots were further incubated with appropriate HRP-coupled secondary antibody (1:2000; Cell Signaling Technology) and visualized by ECL reagent (Thermo 
A

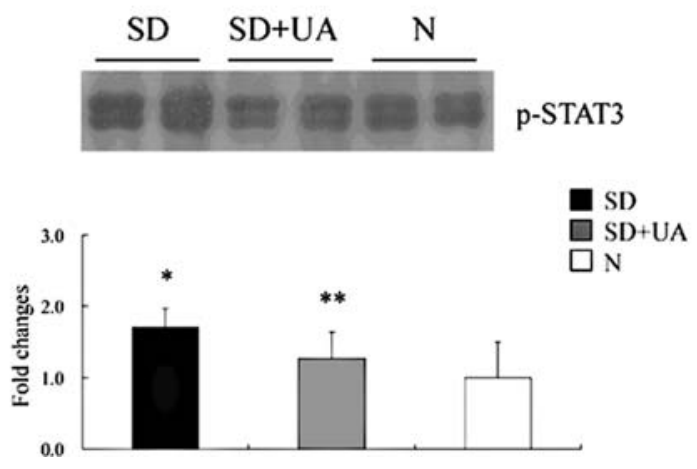

$\mathrm{B}$
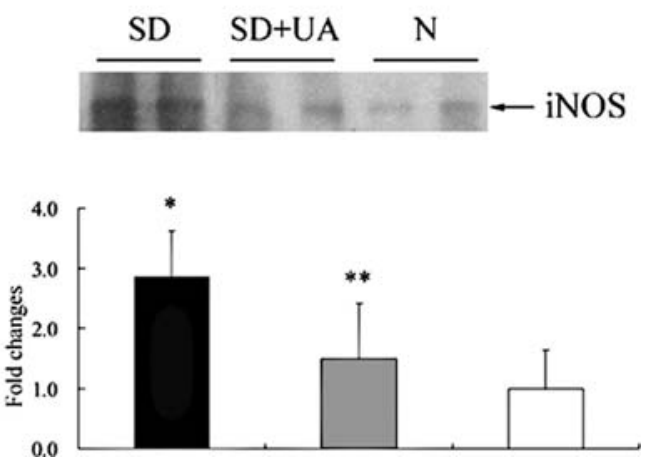

Figure 3. UA inhibits the activation of STAT3 and suppresses the overexpression of iNOS in the kidneys of diabetic mice. (A) Representative Western blot of pho-STAT3tyr705 in renal cortex (top panel) with densitometric quantification results (bottom panel). (B) Representative Western blot of iNOS in renal cortex (top panel), with densitometric quantification results (bottom panel). Expression levels were reported relative to Ponceau $\mathrm{S}$ staining in the same samples, and normalized to nondiabetic group, which was set up as one-fold (SD, diabetes, $\mathrm{n}=5 ; \mathrm{SD}+\mathrm{UA}$, diabetes treated with $\mathrm{UA}$, $\mathrm{n}=5$; $\mathrm{N}$, nondiabetes, $\mathrm{n}=4$; ${ }^{*} \mathrm{p}<0.05$ vs. nondiabetic group; ${ }^{* *} \mathrm{p}<0.05$ vs. diabetic group)

Scientific, Rockland). The developed films were subsequently scanned, and band intensities were quantified with the Quantity One Software (Bio-Rad). The quantified protein levels were further normalized to the nondiabetic group (set as 1).

Statistical analysis. All results were expressed as the mean \pm SD. Statistical significance was evaluated by analyzing data variance with the Kruskal-Wallis test, followed by the Mann-Whitney test. Differences were considered statistically significant at $\mathrm{p}<0.05$.

\section{Results}

Body weight, blood glucose and kidney weight. The values of average body weight, non-fasting blood glucose and kidney weight of experimental animals are summarized in Table I. Consistent with previous reports $(10,13)$, STZ-induced type 1 diabetes not only associated with significant body weight loss and elevated non-fasting blood glucose, but also induced a significant increase in kidney weight in the diabetic group as compared with age-matched nondiabetic controls after 3 months duration of diabetes. Oral administration of low-dose UA $(0.01 \%$ in food $)$ to diabetic mice had no significant effect on any of these characteristics of diabetic mice (Table I).

Glomerular hypertrophy in the kidneys. One of the early morphological changes in the kidneys associated with diabetes is glomerular hypertrophy, resulting from urine filtration overload $(14,15)$. To evaluate the effects of UA treatment on glomerular volume, PASH staining was applied. Our results demonstrated that the average glomerular volume of the diabetic group $(n=10)$ increased by $37 \%$ compared with the nondiabetic controls $(n=10$, Fig. 1). Oral administration of low-dose UA to diabetic mice $(n=10)$ significantly inhibited diabetes-induced glomerular hypertrophy $(\mathrm{p}<0.05$; Fig. 1).

Glomerular type IV collagen accumulation in the kidneys. Accumulation of extracellular matrix proteins in the mesangial space is regarded as the major cause of glomerulosclerosis
(16). Type IV collagen, a non-fibrillar collagen, is one of the major matrix proteins produced by the mesangial cells (17). In the present study, we observed a significant increase in type IV collagen accumulation in glomeruli of the diabetic group compared with the nondiabetic group (three-fold increase vs. nondiabetes; Fig. 2) by semi-quantitative analysis described in Materials and methods. Administration of UA to diabetic mice significantly suppressed such diabetes-induced increase in type IV collagen accumulation in the glomerular area (75\% reduction vs. diabetes; Fig. 2).

Phosphorylated STAT-3 in the kidneys. STAT-3 plays crucial roles in cytokine signaling, energy regulation and inflammation (18). The transcriptional activity of STAT-3 is controlled by the phosphorylation at tyrosine705 of STAT-3, whereas it has been suggested that phosphorylation at serine727 regulates mitochondrial energy metabolism (19). By Western blot analysis, we identified a 1.7-fold increase in phosphorylated STAT-3 at tyrosine705 in the diabetic kidneys compared with nondiabetes (Fig. 3), whereas no effect was observed on the level of phosphorylation at serine727 (data not shown). The administration of UA to diabetic mice not only significantly inhibited a diabetes-induced increase in phosphorylation at tyrosine705 (63\% reduction vs. diabetes; Fig. 3A), but also significantly suppressed phosphorylation of STAT-3 at serine727 in the kidneys (data not shown).

Expression levels of iNOS in the kidneys. Chronic inflammation has been proposed to contribute to the development of DN (16). Western blotting was used to quantify the expression of iNOS, an inflammatory enzyme, in the kidneys of experimental mice. As shown in Fig. 3B, diabetes induced a 2.9-fold increase in iNOS expression compared with the nondiabetic group. The administration of UA to diabetic mice significantly suppressed this diabetes-induced iNOS overexpression in the kidneys (73\% reduction vs. diabetes; Fig. 3B).

Levels of total and phosphorylated (activated) MAPKs in the kidneys. Increasing evidence suggests that MAPKs play important roles in the pathogenesis of diabetic nephropathy 

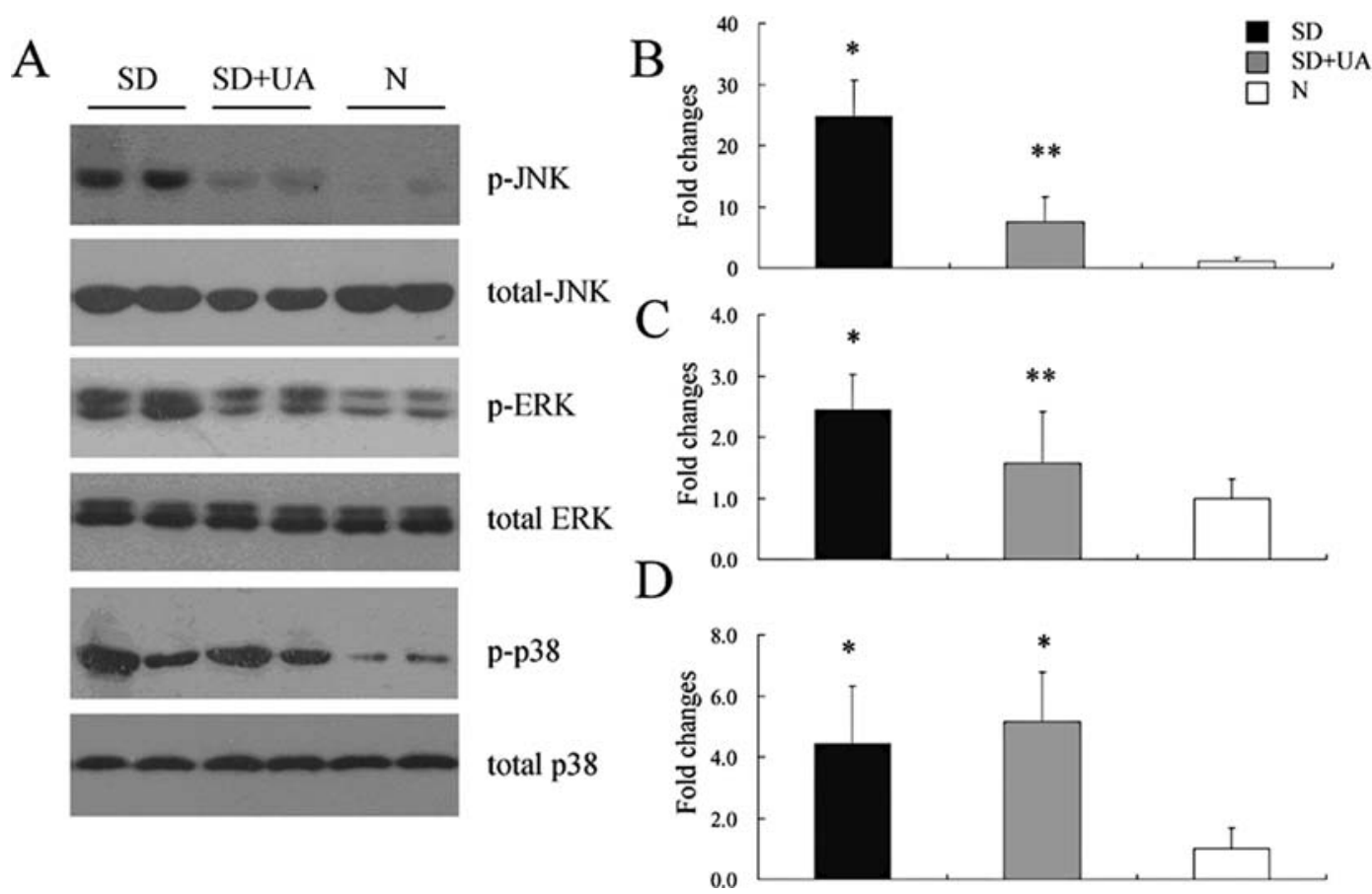

Figure 4. UA inhibits the activations of ERK and JNK, but not the activation of p38 MAPK in the kidneys of diabetic mice. (A) Representative Western blots of pho-JNK, pho-ERK1/2, pho-p38 and their respective total proteins in renal cortex; (B-D) densitometric quantifation results of pho-JNK, pho-ERK1/2 and pho-p38. Expression levels were reported relative to Ponceau S staining in the same samples, and normalized to nondiabetic group, which was set up as one-fold (SD, diabetes, $\mathrm{n}=5$; SD+UA, diabetes treated with $\mathrm{UA}, \mathrm{n}=5 ; \mathrm{N}$, nondiabetes, $\mathrm{n}=4 ;{ }^{*} \mathrm{p}<0.05$ vs. nondiabetic group; ${ }^{* *} \mathrm{p}<0.05$ vs. diabetic group).

(20). MAPK consists of three independent signaling pathways, namely p38 MAPK, ERK1/2 and JNK, which are phosphorylated (activated) by different upstream kinases. Western blotting was used to quantify the total protein levels of JNK, ERK1/2, p38 MAPK and their respective phosphorylated (pho-) forms. As shown in Fig. 4, diabetes induced significant increase in pho-JNK (25-fold), pho-ERK1/2 (2.5-fold) and pho-p38 MAPK (4.5-fold) compared with nondiabetic controls, but showed no effect on the total proteins of JNK, ERK1/2 and p38 MAPK. The administration of UA to diabetic mice had no effect on total proteins of JNK, ERK1/2 and p38 MAPK (Fig. 4). However, UA dramatically suppressed diabetes-induced increases of pho-JNK (73\% reduction vs. diabetes) and pho-ERK1/2 (60\% reduction vs. diabetes), while showed no effect on diabetes-induced increase of pho-p38 MAPK (Fig. 4).

\section{Discussion}

Diabetic nephropathy is one of the major microvascular complications that contribute to the high mortality rate associated with diabetes. In this study, we found that administering UA $(0.01 \%$ in food) to STZ-induced diabetic animals significantly inhibited diabetes-induced early lesions of glomerular hypertrophy and type IV collagen accumulation in glomeruli (Figs. 1 and 2).

We showed that UA markedly inhibited diabetes-induced activation (phosphorylation at tyrosine 705) of STAT-3 in the diabetic kidneys. UA has been shown to inhibit both the constitutive and interleukin-6-inducible STAT-3 activation in myeloma cells (21). Increasing evidence suggests that the activation of the JAK-STAT pathway, especially the activation of STAT-3, plays a critical role in the development of DN (13). For example, the activation of the JAK-STAT pathway has been suggested to contribute to the extracellular matrix accumulation under high glucose condition $(22,23)$; whereas STAT-3 knockdown diabetic mice are markedly lower in proteinuria, mesangial expansion, glomerular cell proliferation and macrophage infiltration than the wild-type diabetic mice via down-regulating the expression of inflammatory markers, the NF- $\mathrm{BB}$ activity and type IV collagen accumulation in the kidneys (13). Similarly, in human glomeruli and tubulointerstitium biopsy samples, the expression levels of STAT-3 are significantly increased in patients with DN than in control subjects (24). Furthermore, medicines used for DN such as captopril (an angiotensin-converting enzyme inhibitor) and candesartan (an angiotensin II receptor antagonist) are believed to exert their therapeutic functions by inhibiting the diabetesinduced activation (phosphorylation) of JAK-2, STAT-1, STAT-3 and STAT-5 in the glomeruli of diabetic rats (25). Thus, inhibition of STAT-3 activation by UA may contribute to the anti-hypertrophic and anti-extracellular matrix accumulation effects of UA in the kidneys of diabetic mice.

Since the STAT family is also involved in the regulation of the inflammation process, which plays an important role in the pathogenesis of DN (26), we next examined the effects of UA on the expression of iNOS, one of key inflammation mediators. We and others have previously reported that iNOS plays critical roles in multiple diabetic complications, including retinopathy, vasculopathy and neuropathy (10, 27-29).

Previous studies on the role of iNOS in DN have shown varied results: on the one hand, increasing iNOS expression 
was identified in the kidneys of diabetic rodents $(30,31)$ similar to that observed by us (Fig. 3B); on the other hand, diabetic iNOS knockout mice showed increased mesangial hypercellularity and increased interstitial type I collagen accumulation compared with the wild-type diabetic mice, possibly due to the complementary overexpression of eNOS in these iNOS knockout mice (32). However, iNOS transgenic mouse has a diabetic phenotype with mild abnormalities in renal morphology (15). Our observations that UA dramatically suppressed the diabetes-induced iNOS overexpression in the kidneys of diabetic mice (Fig. 3B) suggest that UA may also exert its beneficial effects via the inhibition of diabetes-induced iNOS overexpression.

It has been reported that MAPK pathways may co-activate with the JAK-STAT pathway under certain conditions such as angiotensin II stimulation, and affect the development of DN (33). Our results suggest that diabetes induced significant activations of all three MAPK pathways in the renal cortex, as demonstrated by a dramatic increase in the phosphrylated (activated) proteins of p38 MAPK, ERK1/2 and JNK (Fig. 4). The administration of UA inhibited the diabetes-induced activations of ERK1/2 and JNK pathways (Fig. 4), but had no effect on the diabetes-induced increase in the level of phosphorylated p38 MAPK, indicating different MAPK pathway may be responsible for different renal injuries caused by diabetes.

The role p38 MAPK pathway involved in the development of DN has been studied. Lim et al reported that in diabetic MKK $3^{(-/)} \mathrm{db} / \mathrm{db}$ mice (MKK3 is an immediate upstream kinase of p38 MAPK), decreased p38 MAPK signaling in the diabetic kidneys was associated with nephroprotective effects against diabetes-induced renal hypertrophy, podocyte loss, mesangial cell activation, tubular injury and interstitial fibrosis, suggesting MKK3 and perhaps also its downstream p38 MAPK play an important part in the pathogenesis of DN (34). However, our result that UA had no effect on diabetesinduced increase in phosphorylated p38 MAPK suggesting UA does not exert its nephroprotective effects via normalizing the p38 MAPK pathway.

On the other hand, the administration of UA dramatically down-regulated the diabetes-induced activations of ERK1/2 and JNK pathways (Fig. 4). ERK1/2 activation has been suggested to associate with high glucose induced mesangial and tubular cell hypertrophy, TGF- $\beta$ production and fibronectin overexpression $(35,36)$. It was also found in human glomeruli biopsy samples that the mRNA levels of MAPK/ ERK kinase (MEK) 1/2, ERK1/2 and TGF- $\beta$ as well as the protein levels of pho-ERK1/2 were significantly increased at an early stage of DN, as compared with the control subjects (37). The importance of the JNK pathway in renal injury has also been documented: in unilateral ureteral obstruction induced renal fibrosis model, renal fibrosis, collagen IV accumulation and tubular cell death were inhibited in JNK1/2 deficient mice (38).

Overall, our results suggested that UA selectively suppressed diabetes-induced upregulation of ERK1/2 and JNK pathways, but not the p38 MAPK pathway. This observation agrees with the previous study that mesangial cell hypertrophy requires the activations of ERK and JNK pathways, but not the p38 MAPK pathway (39), how UA selectively suppresses the activations of two MAPK pathways still requires further investigation.

In conclusion, oral administration of low-dose of UA to diabetic mice significantly suppressed diabetes-induced lesions of glomerular hypertrophy and excellular matrix accumulation via inhibiting the activation of STAT-3, ERK1/2 and JNK pathways, as well as through inhibiting iNOS overexpression in the diabetic kidneys. Therefore, UA may be further considered as a potential therapeutic agent in inhibiting the progression of DN.

\section{Acknowledgements}

This work was supported by grants from the National Natural Science Foundation of China (NSFC Nos. 30870949, 30801445 and 30970607), the National Basic Research Program of China (2009CB918304), Program for New Century Excellent Talents in University (NECT-10-0623), the Important National Science \& Technology Specific Projects (2009ZX09301-014) and the Key Project of Chinese Ministry of Education (No. 109103).

\section{References}

1. Schena FP and Gesualdo L: Pathogenetic mechanisms of diabetic nephropathy. J Am Soc Nephrol 16 (Suppl 1): 30-33, 2005.

2. Chen S, Jim B and Ziyadeh FN: Diabetic nephropathy and transforming growth factor-beta: Transforming our view of glomerulosclerosis and fibrosis build-up. Semin Nephrol 23: 532-543, 2003 .

3. Forbes JM, Coughlan MT and Cooper ME: Oxidative stress as a major culprit in kidney disease in diabetes. Diabetes 57: 1446-1454, 2008.

4. Soldatos G and Cooper ME: Diabetic nephropathy: Important pathophysiologic mechanisms. Diabetes Res Clin Pract 82 (Suppl 1): 75-79, 2008.

5. Ma FY, Liu J and Nikolic-Paterson DJ: The role of stressactivated protein kinase signaling in renal pathophysiology. Braz J Med Biol Res 42: 29-37, 2009.

6. Lee CH, Wu SL, Chen JC, et al: Eriobotrya Japonica leaf and its triterpenes inhibited lipopolysaccharide-induced cytokines and inducible enzyme production via the nuclear factor-kappaB signaling pathway in lung epithelial cells. Am J Chin Med 36: 1185-1198, 2008

7. Liu J: Pharmacology of oleanolic acid and ursolic acid. J Ethnopharmacol 49: 57-68, 1995.

8. Wang $\mathrm{ZH}$, Hsu CC, Huang CN and Yin MC: Anti-glycative effects of oleanolic acid and ursolic acid in kidney of diabetic mice. Eur J Pharmacol 628: 255-260, 2009.

9. Zheng L, Szabo C and Kern TS: Poly(ADP-ribose) polymerase is involved in the development of diabetic retinopathy via regulation of nuclear factor-kappaB. Diabetes 53: 2960-2967, 2004.

10. Zheng L, Du Y, Miller C, et al: Critical role of inducible nitric oxide synthase in degeneration of retinal capillaries in mice with streptozotocin-induced diabetes. Diabetologia 50: 1987-1996, 2007.

11. Zheng L, Gong B, Hatala DA and Kern TS: Retinal ischemia and reperfusion causes capillary degeneration: Similarities to diabetes. Invest Ophthalmol Vis Sci 48: 361-367, 2007.

12. Ota T, Takamura $\mathrm{T}$, Ando $\mathrm{H}$, Nohara E, Yamashita $\mathrm{H}$ and Kobayashi K: Preventive effect of cerivastatin on diabetic nephropathy through suppression of glomerular macrophage recruitment in a rat model. Diabetologia 46: 843-851, 2003.

13. Lu TC, Wang ZH, Feng X, et al: Knockdown of STAT3 activity in vivo prevents diabetic glomerulopathy. Kidney Int 76: 63-71, 2009.

14. Wei P, Lane PH, Lane JT, Padanilam BJ and Sansom SC: Glomerular structural and functional changes in a high-fat diet mouse model of early-stage type 2 diabetes. Diabetologia 47: 1541-1549, 2004. 
15. Inagi R, Yamamoto $\mathrm{Y}$, Nangaku M, et al: A severe diabetic nephropathy model with early development of nodule-like lesions induced by megsin overexpression in RAGE/iNOS transgenic mice. Diabetes 55: 356-366, 2006.

16. Qian Y, Feldman E, Pennathur S, Kretzler M and Brosius FC III: From fibrosis to sclerosis: Mechanisms of glomerulosclerosis in diabetic nephropathy. Diabetes 57: 1439-1445, 2008.

17. Furness PN: Extracellular matrix and the kidney. J Clin Pathol 49: 355-359, 1996

18. Yu H, Pardoll D and Jove R: STATs in cancer inflammation and immunity: A leading role for STAT3. Nat Rev Cancer 9: 798-809, 2009.

19. Wegrzyn J, Potla R, Chwae YJ, et al: Function of mitochondrial STAT3 in cellular respiration. Science 323: 793-797, 2009.

20. Adhikary L, Chow F, Nikolic-Paterson DJ, et al: Abnormal p38 mitogen-activated protein kinase signalling in human and experimental diabetic nephropathy. Diabetologia 47: 1210-1222, 2004.

21. Pathak AK, Bhutani M, Nair AS, et al: Ursolic acid inhibits STAT3 activation pathway leading to suppression of proliferation and chemosensitization of human multiple myeloma cells. Mol Cancer Res 5: 943-955, 2007.

22. Wang X, Shaw S, Amiri F, Eaton DC and Marrero MB: Inhibition of the Jak/STAT signaling pathway prevents the high glucose-induced increase in TGF-beta and fibronectin synthesis in mesangial cells. Diabetes 51: 3505-3509, 2002.

23. Shi Y, Zhang Y, Wang C, et al: Suppressor of cytokine signaling-1 reduces high glucose-induced tgf-beta1 and fibronectin synthesis in human mesangial cells. FEBS Lett 582: 3484-3488, 2008.

24. Berthier CC, Zhang H, Schin M, et al: Enhanced expression of janus kinase-signal transducer and activator of transcription pathway members in human diabetic nephropathy. Diabetes 58 469-477, 2009.

25. Banes AK, Shaw S, Jenkins J, et al: Angiotensin II blockade prevents hyperglycemia-induced activation of JAK and STAT proteins in diabetic rat kidney glomeruli. Am J Physiol Renal Physiol 286: F653-F659, 2004.

26. Rivero A, Mora C, Muros M, Garcia J, Herrera H and NavarroGonzalez JF: Pathogenic perspectives for the role of inflammation in diabetic nephropathy. Clin Sci 116: 479-492, 2009.

27. Zheng L, Howell SJ, Hatala DA, Huang K and Kern TS: Salicylate-based anti-inflammatory drugs inhibit the early lesion of diabetic retinopathy. Diabetes 56: 337-345, 2007.
28. Vareniuk I, Pavlov IA and Obrosova IG: Inducible nitric oxide synthase gene deficiency counteracts multiple manifestations of peripheral neuropathy in a streptozotocin-induced mouse model of diabetes. Diabetologia 51: 2126-2133, 2008.

29. Zheng L and Kern TS: Role of nitric oxide, superoxide, peroxynitrite and PARP in diabetic retinopathy. Front Biosci 14: 3974-3987, 2009.

30. Fan Q, Liao J, Kobayashi M, et al: Candesartan reduced advanced glycation end-products accumulation and diminished nitro-oxidative stress in type 2 diabetic KK/Ta mice. Nephrol Dial Transplant 19: 3012-3020, 2004.

31. Okada S, Saito M, Kazuyama E, et al: Effects of N-hexacosanol on nitric oxide synthase system in diabetic rat nephropathy. Mol Cell Biochem 315: 169-177, 2008.

32. Trachtman H, Futterweit S, Pine E, Mann J and Valderrama E: Chronic diabetic nephropathy: Role of inducible nitric oxide synthase. Pediatr Nephrol 17: 20-29, 2002.

33. Marrero MB, Fulton D, Stepp D and Stern DM: Angiotensin IIinduced signaling pathways in diabetes. Curr Diabetes Rev 1: 197-202, 2005.

34. Lim AK, Nikolic-Paterson DJ, Ma FY, et al: Role of MKK3p38 MAPK signalling in the development of type 2 diabetes and renal injury in obese $\mathrm{db} / \mathrm{db}$ mice. Diabetologia 52: 347-358, 2009.

35. Al-Rasheed NM, Meakin F, Royal EL, et al: Potent activation of multiple signalling pathways by C-peptide in opossum kidney proximal tubular cells. Diabetologia 47: 987-997, 2004.

36. Lin CL, Wang FS, Kuo YR, et al: Ras modulation of superoxide activates ERK-dependent fibronectin expression in diabetesinduced renal injuries. Kidney Int 69: 1593-1600, 2006.

37. Toyoda M, Suzuki D, Honma M, et al: High expression of PKC-MAPK pathway mRNAs correlates with glomerular lesions in human diabetic nephropathy. Kidney Int 66: 1107-1114, 2004.

38. Ma FY, Flanc RS, Tesch GH, et al: A pathogenic role for c-Jun amino-terminal kinase signaling in renal fibrosis and tubular cell apoptosis. J Am Soc Nephrol 18: 472-484, 2007.

39. Goruppi S, Bonventre JV and Kyriakis JM: Signaling pathways and late-onset gene induction associated with renal mesangial cell hypertrophy. EMBO J 21: 5427-5436, 2002. 